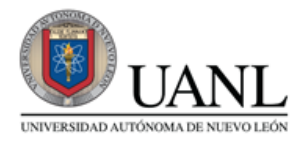

FACPYA
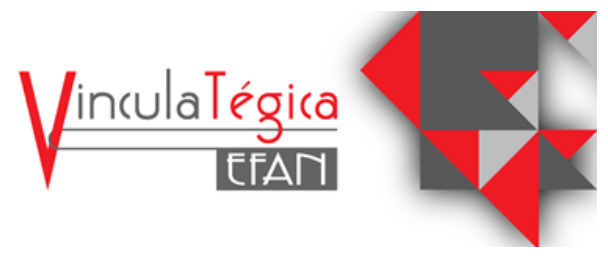

\title{
Determinantes que influyen en el precio de renta de departamentos en Monterrey Nuevo León
}

\author{
Carlos Cruz Lara ${ }^{1}$ \\ ${ }^{l}$ Estudiante de maestría de valuador en la Universidad Autónoma de Nuevo León, \\ carlos.cruzla@uanl.edu.mx, Pedro de Alba S/N, Niños Héroes, Ciudad Universitaria, San Nicolás de los \\ Garza, N.L.
}

Información del artículo revisado por pares

Fecha de aceptación: junio-2021

Fecha de publicación en línea: diciembre-2021

DOI: https://doi.org/10.29105/vtga7.1-117

\begin{abstract}
Resumen
El objetivo de este artículo es indagar sobre los determinantes de los precios mensuales de renta en departamentos que se localizan en Monterrey en los años 2019 y 2020 Nuevo León. La hipótesis es que las personas jóvenes son quienes están en constante búsqueda de departamentos, sobre todo las mujeres ya que buscan estar más cerca de su escuela o de su trabajo. Los datos fueron recabados del periódico -"El Norte"- ya que es un periódico muy La muestra se obtuvo por un mecanismo no probabilístico. Además se utiliza un modelo de precios hedónicos como lo plantea Rosen (1974) para saber cuáles son los aspectos y características que influyen en la toma de decisiones para la búsqueda de departamento. Los principales hallazgos fueron que los departamentos con mayor demanda son los que tienen tres habitaciones, un baño y medio, céntrico y lo más importante que el costo este dentro del rango de los precios del mercado. Finalmente esta investigación se divide en secciones, la primera se refiere a la introducción, la segunda al marco teórico, la tercera al método, la cuarta a los resultados y la quinta a la conclusión.
\end{abstract}

Palabras clave: Departamentos, precios hedónicos, renta, Monterrey Nuevo León, servicios básicos, muestreo no probabilístico.

\begin{abstract}
The objective of this article is to investigate the determinants of the monthly rental prices in apartments that are located in Monterrey in the years 2019 and 2020 Nuevo León. The hypothesis is that young people are the ones who are in constant search of departments, especially women as they seek to be closer to their school or work. The data were collected from the newspaper - "El Norte" - since it is a very newspaper. The sample was obtained by a non-probabilistic mechanism. In addition, a hedonic price model is used as proposed by Rosen (1974) to know what are the aspects and characteristics that influence decisionmaking for department search. The main findings were that the apartments with the highest demand are those with three bedrooms, a bathroom and a half, downtown and the most important thing that the cost is within the range of market prices. Finally, this research is divided into sections, the first refers to the introduction, the second to the theoretical framework, the third to the method, the fourth to the results and the fifth to the conclusion.
\end{abstract}

Keywords: Departments, hedonic prices, rent, Monterrey Nuevo León, basic services, nonprobability sampling.
JEL:
D72,
E25,
I3. 


\section{INTRODUCCIÓN}

No cabe duda que, la adquisición de un departamento en Nuevo León como en cualquier otra parte dota de tranquilidad a las personas por diversas razones por ejemplo: cercanía con el lugar de su trabajo, de centros comerciales, de hospitales, etcétera. Los cuestionamientos que se desea responder son: ¿qué características tienen los departamentos?, ¿por qué la zona influye en su precio?

Es importante aclara que el área metropolitana ha tenido un crecimiento considerable en estos años (2019 - 2020), que ha incrementado su demanda (compra y renta de viviendas), (Chávez. (2006). Asimismo, constituye un problema de infraestructura de la ciudad en cuanto a planeación se refiere, así como equipamiento urbano.

La investigación se realizó del año 2019 al 2020 para poder hacer una comparación sobre los precios de renta y las características de los departamentos antes y después de la pandemia del COVID-19, los datos fueron recabados del periódico "El Norte" para poder llegar a un rango de precios de renta, así como las características de los departamentos. La hipótesis que se quiere probar es identificar si las personas buscan en realidad tener un departamento cómodo a sus necesidades o simplemente estar más cerca de su trabajo. Las características de los departamentos influyen mucho a la hora de elegir un departamento y por lo tanto, son factores que determinan el precio. Cabe resaltar que el muestreo no probabilístico se realizó a 107 personas del área metropolitana de Monterrey ya que lo mínimo eran 100 personas, en el cual se recabó información que veremos representada en tablas y gráficas más adelante.

\section{MARCO TEÓRICO}

La renta de los departamentos siempre ha existido en la actualidad han tenido una mayor demanda debido a que los usuarios decidan rentar un departamento ya sea por la necesidad de estar más cerca del trabajo, de la escuela o simplemente porque no tienen una casa propia. Todo esto se relaciona con las características que buscan en una vivienda desde la década de los setenta se han ido estudiando principalmente para el mercado de las computadoras y televisiones (Michaels, 1979 y Ellickson, 1979).

Algunos autores como Goodman (1978) destacan que los precios de las viviendas son muy variables dependiendo de la zona en la que se localicen; mientras que Witte. Et al., (1979) analiza el precio de la rentabilidad para saber las características de la vivienda ideal que el usuario busca.

Zorrilla (1983) llevó a cabo dicho método (precios hedónicos) en el área metropolitana de Monterrey para confirmar que la calidad de aire y los precios de las viviendas influyen mucho, ya que si una vivienda se localiza en un lugar donde la contaminación es continua, los usuarios no querrán estar en ese lugar por mucho tiempo.

Más que el situar un departamento o una torre departamental como es el caso de la actualidad, es muy importante considerar el aspecto urbano, ya que la ciudad de Monterrey y su área metropolitana no cuenta con una buena distribución urbana y por ende hace más difícil la transportación de un lado a otro, (López. - 2006). Para tener más clara la descripción de precios hedónicos, es una metodología que nos ayuda a saber cuál es el interés del consumidor o del cliente para pagar por un servicio o determinada calidad de vida Mendoza (2009) señala que hay un valor que otorga el entorno, la naturaleza, sus vistas, el vecindario, etcétera. Este valor no solamente le da un valor al objeto o al lugar, sino que también le agrega un valor a la persona. Moreno y Alvarado (2011) destacan que los aspectos que son importantes recalcar son la estructura, el entorno social y la localización. Estas tres características nos describen el departamento; la primera de ellas se refiere al número de recámaras, tamaño del departamento, con terraza, aire acondicionado, servicios de teléfono $\mathrm{y}$ cablevisión, etcétera. La segunda brinda información sobre la seguridad, cercanía de escuelas, tamaño de las familias que viven ahí, etcétera. La tercera nos da información 
de algunos otros aspectos ya que describe la geografía del sitio, su economía, las vistas con las que cuenta, el tráfico al que se enfrenta, etcétera.

\section{MÉTODO}

Realizamos encuestas a usuarios para saber sus preferencias al momento de rentar un departamento, las preguntas base fueron el tamaño del departamento, la razón de porque rentarían, el precio que estarían dispuestos a pagar, la ubicación de este.

Se recabo la información de 107 personas en muestreo no probabilístico en base a una entrevista digital para saber sus características ideales de un departamento, las personas tenían un rango de edad entre los 18 a 60 años.

Se realizó una investigación en la hemeroteca de la biblioteca Alfonsina de la Universidad Autónoma de Nuevo León, los datos se obtuvieron por los meses del año 2019 y 2020 en el periódico el "Norte" para poder analizar las características de los departamentos que estaban publicados y saber los precios dependiendo de la zona en la que se localizaba. Esta búsqueda se centró específicamente en el centro de Monterrey porque ahí es donde se encuentra la mayor fuente de oportunidades para los usuarios. Los resultados obtenidos en la hemeroteca dan a conocer que el mercado de la renta de los departamentos en Monterrey está muy demandado y que casi la mayoría que están publicados tienen las mismas características.

La primera se explica la razón de la encuesta, así como el aclarar que se requería para fines académicos, ya que las personas acceden más fáciles a contestarlas. La segunda sección cuenta con la información general del entrevistado y destacan, datos como edad, género, estado civil, etcétera. Y la última sección representa los aspectos de la rentabilidad de un departamento, aspecto más importante a analizar en dicho artículo.

Inicialmente, se ubica geográficamente Nuevo León y, el municipio de Monterrey, área de estudio y análisis actual, (Imagen 1).

Imagen 1.- Ubicación geográfica del municipio de Monterrey, Nuevo León.

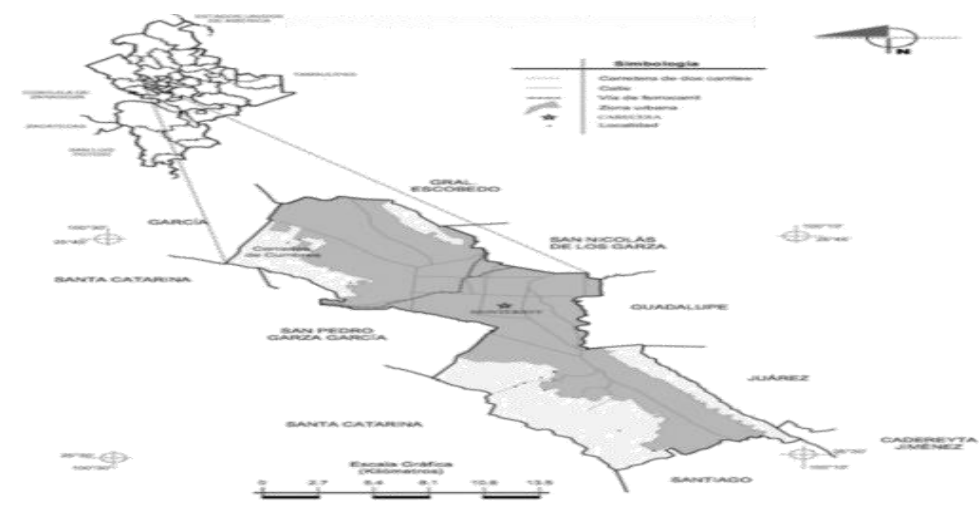

Fuente: INEGI.

Se buscó información sobre departamentos en el centro de Monterrey en el año 2019, y el año 2020 para hacer un comparativo de que tanto se incrementó o se devaluó el precio por la pandemia del COVID-19, así como también determinar las características de los departamentos que estaban en renta entonces respecto de los que se ofrecieron durante la pandemia para verificar los cambios que se han suscitado.

Parte importante de esta investigación es ver cómo el confinamiento ocasionado por el COVID-19 afectó la 
rentabilidad en los departamentos así como identificar las características principales de los mismos en la actualidad y en la zona, siempre considerando, factores como la edad, el género y la importancia de lo que la gente necesita más cuando está buscando un departamento.

La experiencia que el confinamiento ocasionado el COVID-19 hizo que la población pasara mucho tiempo en casa sin poder salir. En ese momento en que las personas valoran correctamente las características antes mencionadas de las viviendas.

\section{RESULTADOS}

De las 107 personas encuestadas se recabó la información con la que se analiza y compara en las siguientes tablas

Tabla 1. Descripción de variables

\begin{tabular}{|c|c|}
\hline Variable. & Descripción. \\
\hline Edad & Muestra la edad en forma numérica del encuestado \\
\hline Género & Variable dummy: 1=Hombre, 2=Mujer \\
\hline Civil & Estado civil en el que se encuentra el entrevistado \\
\hline Escolaridad & $\begin{array}{l}\text { Formación académica, como último nivel de estudio } \\
\text { completado }\end{array}$ \\
\hline Ingresos & Ingreso salarial por mes \\
\hline Ubicación & $\begin{array}{l}\text { Cerca de qué área tiene preferencia por encontrar } \\
\text { departamento }\end{array}$ \\
\hline Recámaras & $\begin{array}{l}\text { Cantidad de recámaras busca tener en su } \\
\text { departamento de renta }\end{array}$ \\
\hline Baños & Cantidad de baños que desea cuente el departamento \\
\hline Amueblado & $\begin{array}{l}\text { Si el entrevistado desea conseguir un departamento } \\
\text { amueblado. } \\
\text { Variable dummy: } 1=\mathrm{S} \text { í, } 2=\mathrm{No}\end{array}$ \\
\hline Mensualidad & $\begin{array}{l}\text { Pagos mensuales en pesos, que está dispuesto a pagar } \\
\text { por un departamento amueblado }\end{array}$ \\
\hline Sin muebles & $\begin{array}{l}\text { Razón por la cual no busca un departamento } \\
\text { amueblado }\end{array}$ \\
\hline Servicios & Servicios que necesita en su departamento \\
\hline Cochera & $\begin{array}{l}\text { Si el entrevistado requiere cochera. } \\
\text { Variable dummy: } 1=\mathrm{S} 1 \text {, } 2=\mathrm{No}\end{array}$ \\
\hline T.auto & Tamaño del automóvil que el encuestado utiliza \\
\hline S.cochera & El entrevistado no desea contar con cochera \\
\hline Autos & Cantidad de automóviles que tiene el entrevistado \\
\hline Departamento & Tamaño del departamento en m2 que busca \\
\hline Patio & $\begin{array}{l}\text { Si el entrevistado quiere patio en su departamento. } \\
\text { Variable dummy: } 1=\text { Sí, } 2=\text { No }\end{array}$ \\
\hline T.patio & Tamaño del patio que requiere \\
\hline C.patio & Área por la que desea cambiar el patio \\
\hline Terraza & $\begin{array}{l}\text { Si el entrevistado quiere terraza. Variable dummy: } \\
1=\text { Sí, } 2=\text { No }\end{array}$ \\
\hline T.ideal & Características de la terraza ideal del entrevistado \\
\hline C.terraza & Área por la que desea cambiar la terraza \\
\hline Vistas agradables & $\begin{array}{l}\text { Si el entrevistado prefiere vistas agradables. } \\
\text { Variable dummy: } 1=\text { Sí, } 2=\text { No }\end{array}$ \\
\hline D.vistas agradables & $\begin{array}{l}\text { Descripción de vistas agradables que considera el } \\
\text { entrevistado }\end{array}$ \\
\hline
\end{tabular}


\begin{tabular}{cl} 
C.vistas agradables & $\begin{array}{l}\text { Cambiar vistas agradables por otro aspecto a beneficio } \\
\text { personal }\end{array}$ \\
\hline Fuente: Elaboración propia.
\end{tabular}

La Tabla 2 se explica la frecuencia y el porcentaje a estudiar de solo algunas variables.

Con estas logramos sacamos la media y la moda para identificar en nuestros encuestados si predominan más hombres o mujeres, así como otras variables como el ingreso salarial, su escolaridad, estado civil, y características especiales del departamento.

De esta manera, los resultados son acordes con lo que establece Zorrilla (1983), es decir, que los precios van de acuerdo a las características que buscan las personas y de las prioridades que les asignan en su bienestar. Dicho análisis se verá interpretado en las siguientes tablas.

Tabla 2. Variables sociodemográficas de los encuestados y porcentajes

\begin{tabular}{lll}
\hline Variable & Frecuencia & Porcentaje \\
\hline Edad & & \\
\hline Menos de 25 & 29 & 26.6 \\
De 26 a 35 & 44 & 40.3 \\
De 36 a 50 & 15 & 13.7 \\
De 51 o mas & 17 & 15.5 \\
No contestaron & 2 & 3.9 \\
Total & 107 & 100 \\
\hline Género & & \\
\hline Hombre & 42 & 39.3 \\
Mujer & 65 & 60.7 \\
Total & 107 & 100.0 \\
\hline Estado civil & & \\
\hline Soltero(a) & 56 & 52.3 \\
Casado (a) & 47 & 43.9 \\
Separado (a) & 1 & 0.9 \\
Divorciado (a) & 3 & 2.8 \\
Total & 107 & 100 \\
\hline Escolaridad & & \\
\hline Secundaria & 2 & 1.9 \\
Secundaria o técnica & 26 & 24.3 \\
Licenciatura & 66 & 61.7 \\
Maestría & 13 & 12.1 \\
Total & 107 & 100 \\
\hline Ingresos & 9 & 31.8 \\
\hline Menos de 5 mil & 38 & \\
De 5 a 10 mil & 26 & \\
De 10 a 15 mil & 34 & \\
Más de 15 mil & & \\
\hline & & \\
\hline
\end{tabular}




\begin{tabular}{|c|c|c|}
\hline Total & 107 & 100 \\
\hline
\end{tabular}

La mayoría de los encuestados fueron mujeres de 26 a 35 años, solteras, con una licenciatura y un ingreso de 5 a 10 mil pesos mensuales. Estos resultados coinciden con la hipótesis de la investigación de la cual es que las personas jóvenes son quienes están en constante búsqueda de departamentos y más las mujeres ya que buscan estar más cerca de su escuela o de su trabajo.

Los otros datos que salieron en la tabla nos dicen que hay una población mínima que tiene estudios superiores a la licenciatura (12\%) y que tienen un ingreso mensual mayor, esto quiere decir que solo estas personas pueden adquirir de un departamento más costoso y con más virtudes para darle valor agregado.

Tabla 3. Estadísticos descriptivos de las variables.

\begin{tabular}{|l|c|c|c|c|}
\hline & Mínimo & Máximo & Media & $\begin{array}{c}\text { Desviación } \\
\text { estándar }\end{array}$ \\
\hline Ubicación & 1 & 3 & 2.18 & 0.450 \\
\hline Recámaras & 1 & 3 & 2.10 & 0.640 \\
\hline Baños & 1 & 3 & 2.35 & 0.765 \\
\hline Amueblado & 1 & 2 & 1.56 & 0.498 \\
\hline Mensualidad & 1 & 3 & 1.71 & 0.621 \\
\hline Sin muebles & 1 & 3 & 2.43 & 0.640 \\
\hline Servicios & 1 & 3 & 2.14 & 0.848 \\
\hline Cochera & 1 & 2 & 1.21 & 0.411 \\
\hline T.auto & 1 & 3 & 1.43 & 0.805 \\
\hline S.cochera & 1 & 3 & 1.47 & 0.784 \\
\hline Autos & 1 & 4 & 2.00 & 0.749 \\
\hline Departamento & 1 & 3 & 2.15 & 0.639 \\
\hline
\end{tabular}

Fuente: Elaboración propia

En la Tabla 3 nos damos cuenta de las estadísticas de cada una de las variables para considerar cual es la que más utilizan y a base de estos resultados saber cuáles características son las más buscadas por las personas. Ya que hay muchos factores que afectan en la toma de decisión para la busca de un departamento.

La importancia de hacer estas investigaciones es demostrar cuáles son los departamentos con mayor demanda y a base de esta información las desarrolladoras puedan hacer el departamento para que se les rente lo antes posible.

Con esta tabla nos basamos también para identificar las características que en cierta manera son importantes, como son las vistas, el entorno, la contaminación ambiental, etc. Así como lo explica Saphores y Aguilar (2005) que el precio afecta mucho por los malos olores que está en la zona, ya que si está cerca de una industria, siempre tendrá malos olores y afectará al precio del departamento.

López Rizzo (2006) menciona sobre qué tan importante es la zona en la que se ubica el departamento, ya que si es una zona en donde no tienes fácil acceso a carreteras o tienes problemas para transportarte a un lugar a otro esto le resta de igual forma el costo al departamento, por eso en la tabla se ve que un factor muy importante y que muchas personas escogieron era que 
estuviera cerca de su trabajo.

Gráfica 1. Tamaño adecuado del departamento para los encuestados

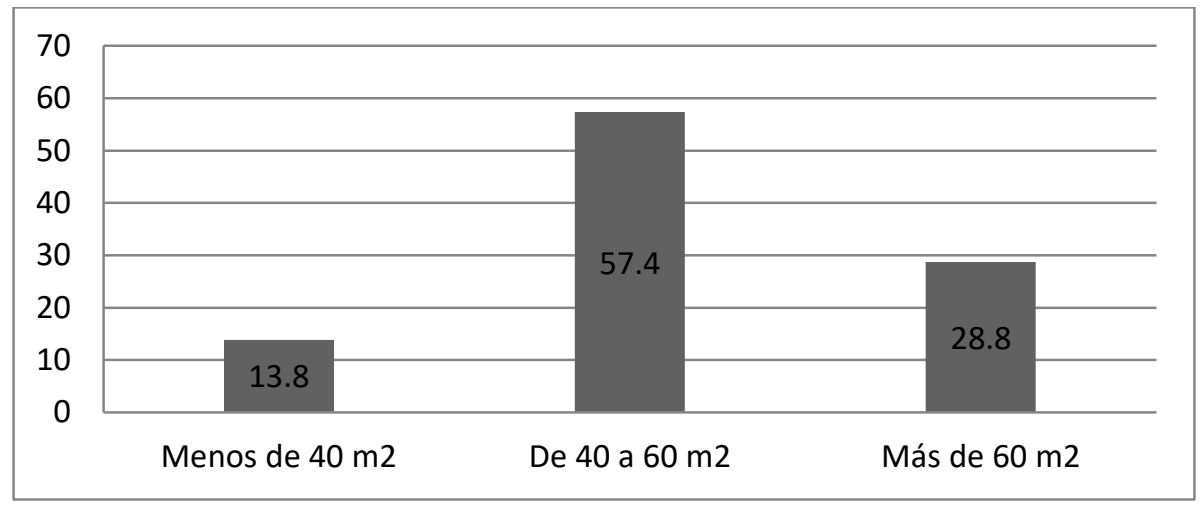

Fuente: Elaboración propia.

La Gráfica 1 indica que más de la mitad de los encuestados prefieren tener un departamento de 40 a $60 \mathrm{~m}^{2}$.

Este dato se vio mucho en la investigación preliminar que se realizó en la hemeroteca de la biblioteca Alfonsina en donde los departamentos publicados tenían estas medidas y estaban en un costo común a los demás departamentos del mercado, lo que variaba eran los muebles y los servicios.

Mientras que el $29 \%$ de los encuestados nos indican que hay personas que si quieren un departamento amplio en donde puedan tener más áreas. El porcentaje restante $13.8 \%$ prefieren tener un departamento pequeño quizás sea por tener un costo menor o por el simple hecho de que no se la pasa mucho tiempo en el departamento. Después de analizar esta gráfica y saber la medida estándar del departamento, pasaremos a analizar los siguientes gráficos para poder llegar a una conclusión con los datos recabados.

Gráfica 2. Porcentaje de entrevistados que desean patio

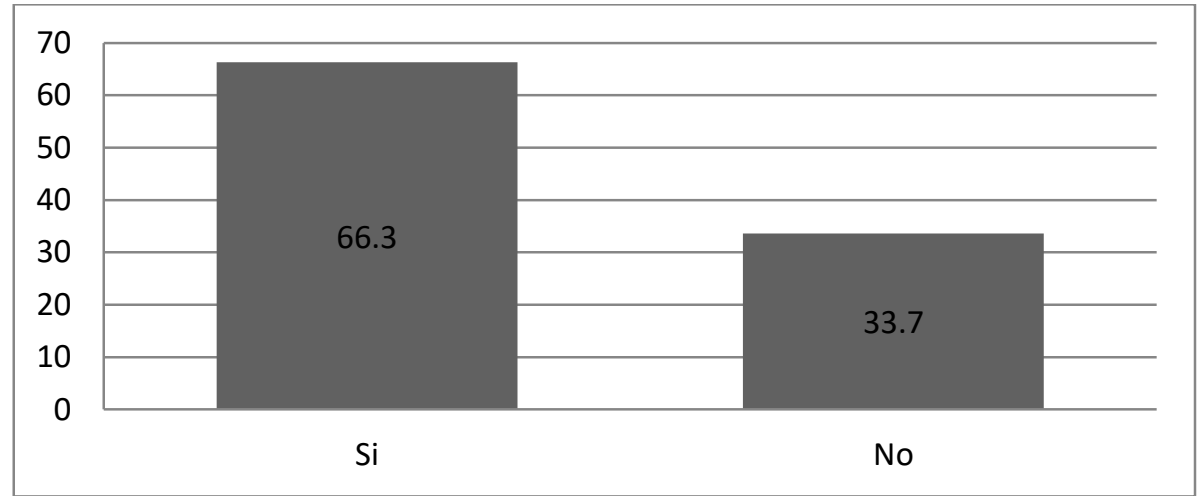

Fuente: Elaboración propia.

En la Gráfica 2 el $66 \%$ nos indicó que esta dentro de sus necesidades el conseguirlo con patio, mientras que el $33.7 \%$ indica que no quieren patio en el departamento que buscan ya que la causa principal es que no pasan mucho tiempo en casa. Podemos deducir que la mayoría de los entrevistados desean un área al exterior de su hogar en el que puedan tener algún tipo de recreación. De las personas que 
quieren tener el patio en su departamento el porcentaje mayor respondió que desean un patio de tamaño mediano, como se aprecia en la Gráfica 3, con el 59\% mientras que el $11 \%$ desea un espacio grande. Y solo el $29 \%$ respondió que quería solo un espacio pequeño. Se puede deducir con estas respuestas que esperan que el patio tenga significancia en el departamento de búsqueda.

Gráfica 3. Tamaño de patio que buscan los encuestados

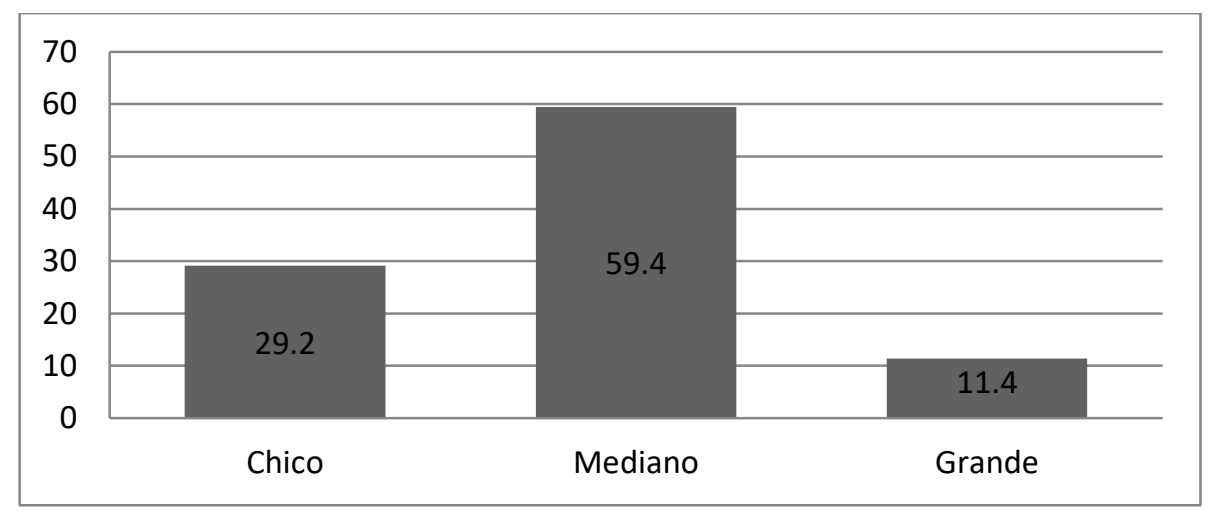

Fuente: Elaboración propia.

La Gráfica 4 retoma el resultado de las personas que no desean tener patio, es decir el $33.7 \%$ de los entrevistados. Estas personas desean cambiar esta área por alguna otra que sea más de su aprovechamiento, se expusieron tres posibles respuestas a lo que ellos respondieron que preferirían tener una recámara en lugar de ese patio, con el 36\% de los encuestados. Seguidos por una cocina, reconociendo que se refieren a tener una cocina más amplia ya que usualmente en los espacios que rentan como departamentos tienden a tener espacios muy reducidos. $\mathrm{Y}$ al final con un $31.5 \%$ indican que preferirían tener un espacio mejor adaptable a lo que es la sala, pensando que estos encuestados son más del ámbito social por lo cual requieren más espacio en esta área del departamento.

Gráfica 4. Espacio que desean aprovechar en lugar de un patio

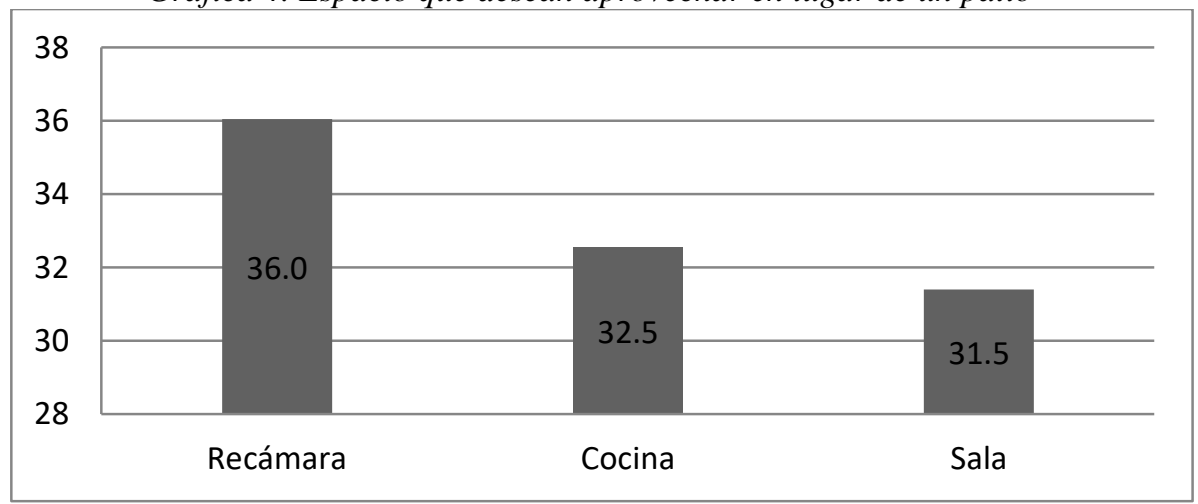

Fuente: elaboración propia.

A continuación, se analiza la percepción de una terraza, esto 
considerando que existen departamentos que se ofrecen en una segunda planta y se presta a tener esta área, de los entrevistados el $64.7 \%$ prefieren tener una terraza, considerando que el centro de Monterrey contiene vistas a los mejores cerros, y una terraza en esta zona pudiera ser de lo más aprovechable. Mientras que el $35.3 \%$ no quisiera tener una terraza en su departamento de búsqueda. Considerando las personas que prefieren terrazas, se les cuestiono por las vistas que prefieren tener al estar en sus terrazas, dejando como opción a elegir tres, y el porcentaje que más se vio favorecida, son las vistas agradables con el $53.1 \%$, por lo cual se puede deducir que las personas que desean una terraza es por esta condición, que necesitan tener vistas que puedan aprovechar en ella. Seguido por espacios abiertos con el $35.4 \%$ considerando que las terrazas son más para tomar el aire libre. Y por último con el $11.5 \%$ requieren una terraza lo más privada y personal posible, véase la Gráfica 6 a continuación.

Gráfica 5. Porcentaje de entrevistados que desean terraza

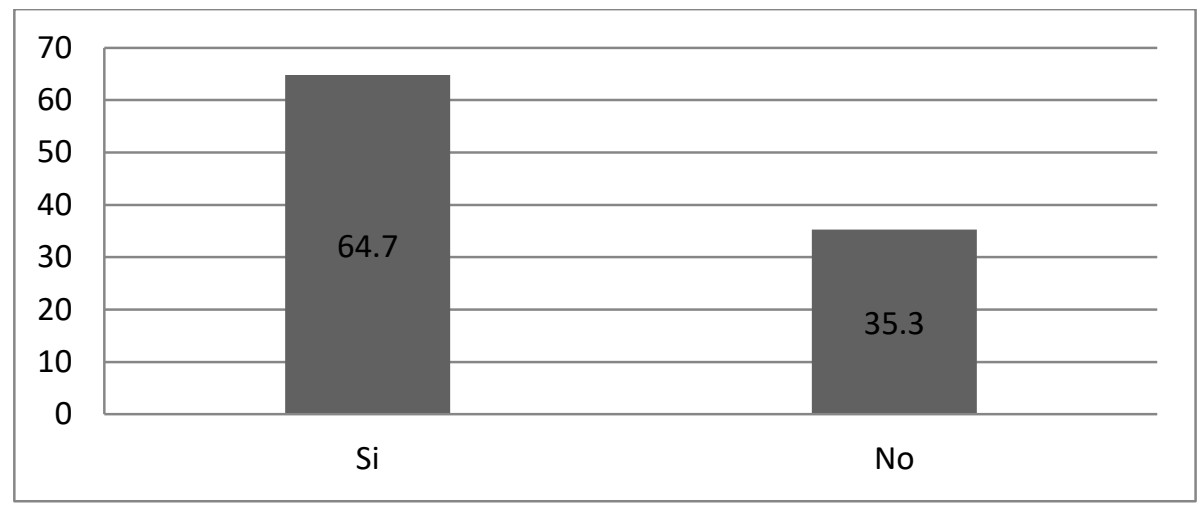

Fuente: Elaboración propia.

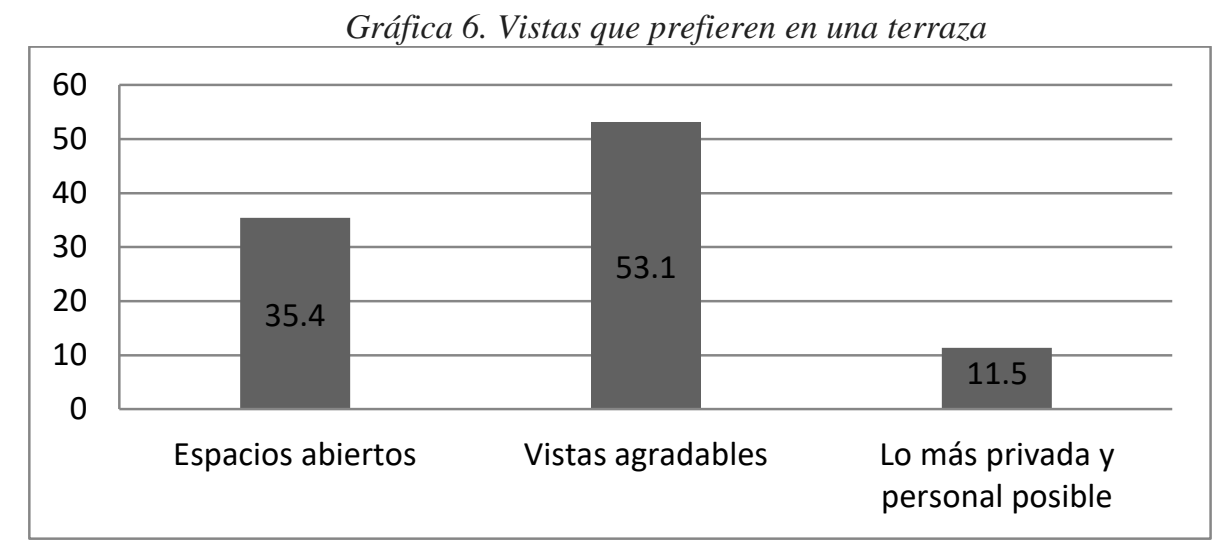

Fuente: Elaboración propia.

A las personas que no quieren una terraza, se les cuestionó que área prefieren en lugar de una terraza, enlistando tres posibilidades se reportó que la recámara obtuvo el $40 \%$ de las preferencias, seguido 
por una sala de juegos con el $32 \%$ y al final con un estudio de trabajo con el 28\%. Considerando que de personas que no quieren terrazas solo obtuvimos el $35.2 \%$, se podría decir que aproximadamente una tercera parte de los entrevistados no la quiere. Estos porcentajes se describen en la Gráfica 7.

Gráfica 7. Área que prefieren en lugar de una terraza

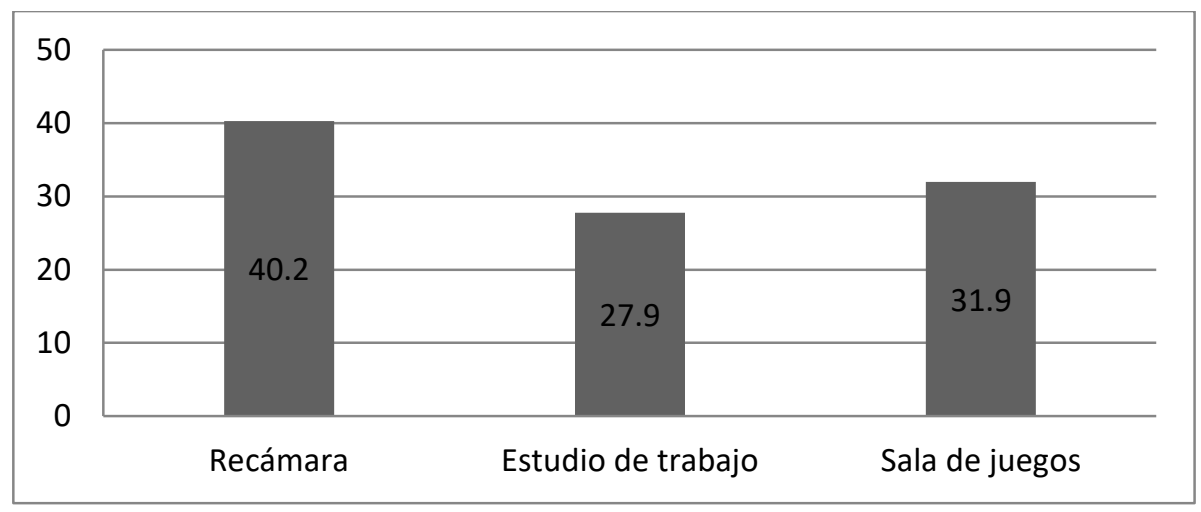

Fuente: Elaboración propia.

También se busca tener la percepción del encuestado en cuanto a que se refieren ellos con las vistas agradables, por lo cual una de las preguntas era ver las consideraciones que ellos tenían o el concepto al que se referían al hablar de vistas agradables, lo que se describen la Gráfica 8, donde la respuesta con mayor porcentaje fue vistas a los cerros, con un
$64.6 \%$, donde comprobamos una vez mas que es uno de los detonantes o cosas principales a la hora de seleccionar un departamento, seguido de vistas a un jardín considerando que fueran en una sola planta con un $30.3 \%$ y por ultimo con un $5.1 \%$ vistas privadas adornadas por el usuario, como se describe en la Gráfica 8

Gráfica 8. Que considera una vista agradable el encuestado

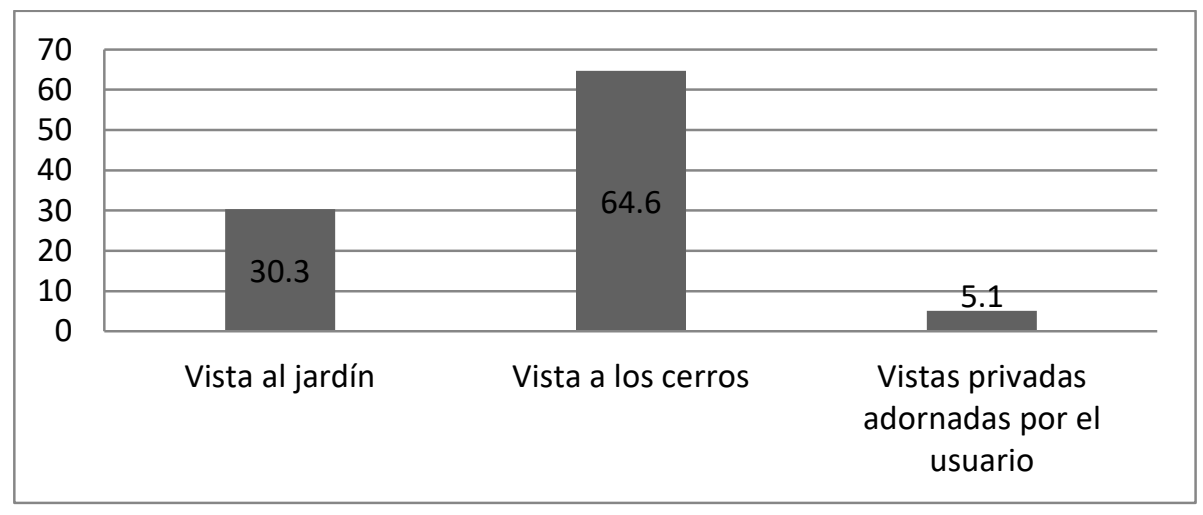

Fuente: Elaboración propia.

En la Gráfica 9, se cuestionó las personas que no quieren unas vistas agradables, cuál sería la opción que tendría mayor peso, al buscar departamento si no contara con estas vistas agradables, o fueran otros conceptos de su agrado. Las principales respuestas con un 38\% destacan dos espacios 
adecuados y zonas privilegiadas de accesos rápidos.

Gráfica 9. Que prefiere en lugar de vistas agradables

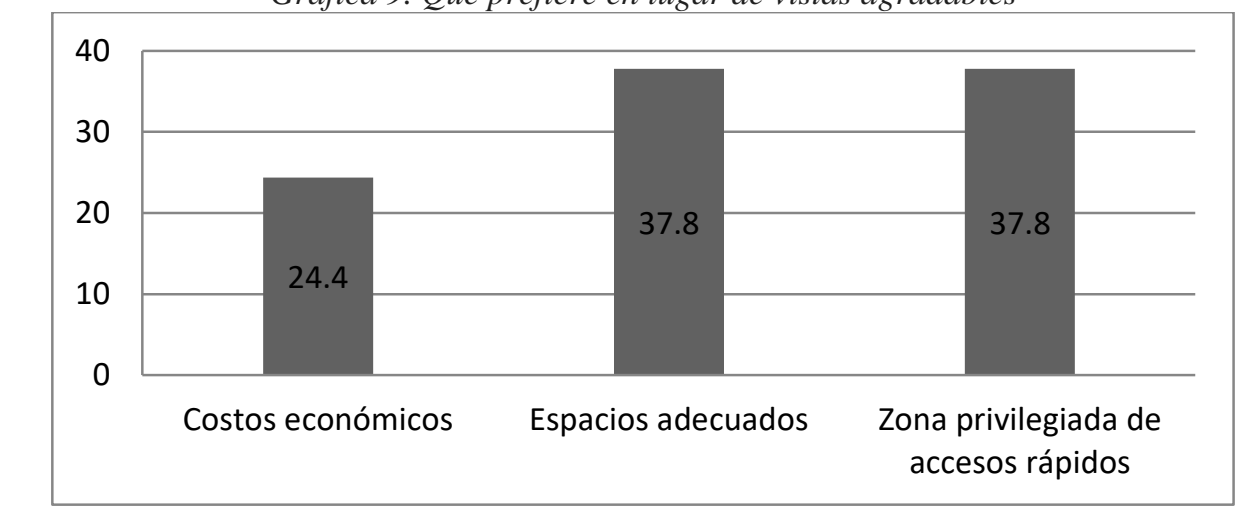

Fuente: Elaboración propia.

\section{CONCLUSIONES}

Los departamentos están teniendo una gran demanda en el mercado por la sociedad que busca estar cerca de su empleo y de sus estudios, es importante abordar el tema de identificar cuáles son las características que más buscan las personas para rentar.

Lo más importante de este estudio es que las personas puedan rentar un departamento con mayor facilidad ya teniendo en cuenta las características primordiales.

No es el simple hecho de construir y ya, es resolver todos los problemas que se van a presentar, ya sea para la sociedad y también para los usuarios que vayan a rentar un departamento; me refiero a que si no se hace una buena planeación en lo urbano para poder llegar bien al lugar, el sitio no tendrá una buena aceptación por la sociedad porque su transcurso de llegada será difícil, en cambio sí se planea bien la infraestructura urbana, es más probable que tenga una gran aceptación por la sociedad y por supuesto que el precio incrementaría.

Podemos decir que los departamentos se rigen por niveles, el básico es el que todos buscan, el medio es aquel que no todos pueden tener y el alto es aquel que cumple con características muy específicas de personas que tienen la posibilidad de adquirirlo y poder sustentar las renta y el mantenimiento. Hay mercado para todos y es bueno saber los factores que esto lo constituyen.

Sin dejar de lado que en estos tiempos estamos batallando con la economía del país por el COVID-19 tenemos que estar de acuerdo en que no siempre se podrá tener un costo elevado, es buscar un precio "justo" y rentar a ese precio porque de otra manera no podrás rentar los departamentos.

Para el descanso y el confort no hay precio que no valga pero también hay que estar conscientes de cuánto tiempo tendrías que invertir para lograr alcanzar tener un departamento soñado. Hoy en día estamos aprendiendo a valorar lo mucho o poco que tenemos y así ser felices, ya que si hay posibilidad de tener un departamento muy bien ubicado, con buenas vistas, amplio, etcétera es a base de seguir luchando por salir adelante y sin importar este trago amargo que nos trajo la pandemia del COVID-19. 


\section{REFERENCIAS}

Chávez Alvarado, Rosalía (2006), "Monterrey: una revisión metropolitana”, en Mario Bassols Ricardez y Roberto Garciìa Ortega (Coords.), Explorando el régimen urbano en México. Un análisis metropolitano, México: El Colegio de la Frontera Norte - Universidad Autoìnoma Metropolitana, Unidad Iztapalapa - Plaza y Valdeìs.

Goldman, Fredy Michael Grossman (1978), "The demand for pediatric care: anhedonic approach", en The Journal of Political Economy, vol. 86, núm. 2, parte 1, Chicago IL: University Of Chicago

López Rizzo, Humberto César (2006), Valuación de las características de la vivienda del AMM mediante la metodología de precios hedónicos, tesis de maestría en Economía, Monterrey, México: UANL. Facultad de Economía

Mendoza González, Gabriela (2009), Análisis de cambio de uso de suelo y sus implicaciones en la prestación de servicios ecosistémicos en la costa de Veracruz, tesis de maestría, Xalapa, Veracruz: Instituto de Ecología.

Michaels, Robert (1979), "Hedonic prices and the structure of the digital computer industry", en The Journal of Industrial Economics, vol. 27, núm. 3, New Jersey: Wiley Blackwell.

Moreno, R. y Alvarado, E. (2011). El entorno social y su impacto en el precio de la vivienda: un análisis de precios hedónicos en el área metropolitana de Monterrey. Trayectorias, (14)33, 131-147.

Saphores, Jean-Daniel y Ismael Aguilar (2005), "Smelly local polluters and residential property values: an hedonic analysis of four Orange County (California) cities", en Estudios Económicos, vol. 20, núm. 2, México: El Colegio de México.

Witte, Ann D., Howard Sumka and Homer Erekson(1979), "An estimate of a structural hedonic price model of the housing market: an application of Rosen's theory of implicit markets", en Econometríca, vol. 47, núm. 5, New York: The Econometric Society.

Zorrilla Bustamante, Alicia (1983), Precios implícitos de las características de la vivienda en el AMM, tesis de licenciatura en Economía, Monterrey, México: UANL, Facultad de Economía. 